Doriana Landi, MD, PhD*

Nicola De Rossi, MD*

Sara Zagaglia, MD

Cristina Scarpazza, PhD

Luca Prosperini, MD,

$\mathrm{PhD}$

Maria Albanese, MD,

$\mathrm{PhD}$

Fabio Buttari, MD

Francesco Mori, MD, $\mathrm{PhD}$

Girolama Alessandra Marfia, MD, PhD

Maria Pia Sormani, PhD $\ddagger$

Ruggero Capra, MD $\ddagger$

Diego Centonze, MD, $\mathrm{PhD} \ddagger$

On behalf of the Italian PML study group

Correspondence to

Dr. Capra:

ruggero.capra@gmail.com

Editorial, page 1108

Supplemental data at Neurology.org

\section{No evidence of beneficial effects of plasmapheresis in natalizumab-associated PML}

\section{全}

\section{ABSTRACT}

Objective: To examine retrospectively the effects of plasmapheresis (PLEX) on the survival and clinical outcomes of patients with multiple sclerosis (MS) and natalizumab (NTZ)-associated progressive multifocal leukoencephalopathy (PML).

Methods: The medical literature was searched for the terms natalizumab and progressive multifocal leukoencephalopathy. A total of 193 international and 34 Italian NTZ-PML cases were included. Clinical outcome was determined by comparing the patients' clinical status at PML diagnosis with status after PML resolution. The effects on survival and clinical outcome of PLEX, sex, age, country, pre-PML Expanded Disability Status Scale score, NTZ infusion number, prior immunosuppressant exposure, PML symptoms, PML lesion location at diagnosis, CSF JC virus status and copies, additional PML treatments and steroids, and PML immune reconstitution inflammatory syndrome (IRIS) development were investigated with both univariate and multivariate analyses.

Results: A total of 219 NTZ-PML cases were analyzed, and 184 (84\%) underwent PLEX, which did not reduce the mortality risk or the likelihood of poor vs favorable outcomes. Country was predictive of mortality and poor outcome, while PML-IRIS development was predictive of poor outcome.

Conclusions: PLEX did not improve the survival or clinical outcomes of Italian or international patients with MS and NTZ-PML, suggesting that this treatment should be performed cautiously in the future.

Classification of evidence: This study provides Class III evidence that for patients with NTZ-PML, PLEX does not improve survival. The study lacks the statistical precision to exclude an important benefit or harm of PLEX. Neurology ${ }^{\circledR}$ 2017;88:1144-1152

\section{GLOSSARY}

EDSS = Expanded Disability Status Scale; HR = hazard ratio; IRIS = immune reconstitution inflammatory syndrome; JCV = JC virus; $\mathbf{M S}=$ multiple sclerosis; $\mathbf{N T Z}$ = natalizumab; PLEX = plasmapheresis; PML = progressive multifocal leukoencephalopathy; ROW = rest of the world.

Natalizumab (NTZ) treatment in patients with multiple sclerosis (MS) is associated with an increased risk of the development of progressive multifocal leukoencephalopathy (PML), an opportunistic infection caused by the JC virus (JCV). As of March 4, 2016, 635 cases of NTZ-associated PML (NTZ-PML) have been confirmed in patients with MS in the postmarketing setting (hcp.biogen-international.com/tysabri_update.aspx?ID=22076). No specific treatments are available, and the NTZ-PML mortality rate is about $24 \%$ (hcp.biogeninternational.com/tysabri_update.aspx? ID = 22076). ${ }^{1}$ Older age, high pre-PML disability, high number of CSF JCV copies, MRI findings consistent with multifocal damage, and worse Karnofsky Performance Scale scores at the time of PML diagnosis are predictive of poor

\footnotetext{
*These authors contributed equally to this work.

$\ddagger$ These authors contributed equally to this work as senior authors.

From the Multiple Sclerosis Clinical and Research Unit, Department of Systems Medicine (D.L., M.A., F.B., F.M., G.A.M., D.C.), Tor Vergata University, Rome; IRCCS Istituto Neurologico Mediterraneo (INM) Neuromed (D.L., M.A., F.B., F.M., G.A.M., D.C.), Pozzilli; Regional Multiple Sclerosis Center (N.D.R., C.S., R.C.), ASST-Spedali Civili di Brescia, Montichiari; Neurological Clinic (S.Z.), Marche Polytechnic University, Ancona; Department of Neurology and Psychiatry (L.P.), Sapienza University, Rome; and Biostatistics Unit, Department of Health Sciences (DISSAL) (M.P.S.), University of Genoa, Italy.

Coinvestigators of the Italian PML study group are listed at Neurology.org.

Go to Neurology.org for full disclosures. Funding information and disclosures deemed relevant by the authors, if any, are provided at the end of the article.
} 
Table 1 Descriptive characteristics of the population

\begin{tabular}{|c|c|c|c|}
\hline & $P L E X+(n=184)$ & PLEX- ( $n=35)$ & p Value \\
\hline Sex, n (\%) & & & $0.02^{\mathrm{a}}$ \\
\hline Male & $61(33)$ & $4(11)$ & \\
\hline Female & $123(67)$ & $31(89)$ & \\
\hline Age at PML diagnosis, $y$, mean $( \pm S D)^{b}$ & $43.3( \pm 8.9)$ & $40.9( \pm 10.7)$ & 0.26 \\
\hline Country, n (\%) & & & 0.36 \\
\hline Europe & $136(74)$ & 29 (83) & \\
\hline US and ROW & $48(26)$ & $6(17)$ & \\
\hline Pre-PML EDSS, median (range) ${ }^{c}$ & $3.5(0-7.5)$ & $3.5(1.0-7.0)$ & 0.64 \\
\hline No. of NTZ infusions, mean $( \pm S D)^{d}$ & $31.9( \pm 12.7)$ & $35.9( \pm 14.8)$ & 0.10 \\
\hline Prior immunosuppressants, $\mathrm{n}(\%)$ & & & 0.09 \\
\hline Yes & $38(36)$ & $5(17)$ & \\
\hline No & $68(64)$ & $24(83)$ & \\
\hline $\begin{array}{l}\text { EDSS at PML diagnosis, } \\
\text { median }\left(\text { range) }^{\mathrm{e}}\right.\end{array}$ & $5.0(0-9.0)$ & $4.0(2.0-8.0)$ & 0.62 \\
\hline Symptoms at PML diagnosis, $n(\%)$ & & & 0.60 \\
\hline Yes & 95 (92) & 27 (87) & \\
\hline No & $8(8)$ & $4(13)$ & \\
\hline $\begin{array}{l}\text { PML lesion localization at } \\
\text { diagnosis, } \mathrm{n}(\%)\end{array}$ & & & $0.04^{a}$ \\
\hline Supratentorial & $69(87)$ & $17(68)$ & \\
\hline Infratentorial & $6(7)$ & $2(8)$ & \\
\hline Both & 5 (6) & $6(24)$ & \\
\hline CSF JCV status at diagnosis, $\mathrm{n}(\%)$ & & & 0.25 \\
\hline Positive & $160(88)$ & $27(79)$ & \\
\hline Negative & $21(12)$ & $7(21)$ & \\
\hline $\begin{array}{l}\text { CSF JCV copies/mL (anytime), } \\
\text { median (interval) }^{f}\end{array}$ & $340(0-4,831,575)$ & $57(0-26,300)$ & $0.001^{a}$ \\
\hline Additional treatments, $\mathrm{n}(\%)$ & & & $0.002^{a}$ \\
\hline None & 35 (38) & $23(72)$ & \\
\hline Mefloquine or mirtazapine & $56(62)$ & 9 (18) & \\
\hline PML-IRIS development, n (\%) & & & 0.99 \\
\hline Yes & $92(81)$ & $24(83)$ & \\
\hline No & $21(19)$ & $5(17)$ & \\
\hline $\begin{array}{l}\text { PML diagnosis-IRIS interval, } d \text {, } \\
\text { median (interval) }\end{array}$ & $27.5(0-90)$ & $45(6-120)$ & 0.14 \\
\hline Treatment with steroids, $\mathrm{n}(\%)$ & & & 0.99 \\
\hline Yes & $72(80)$ & $26(81)$ & \\
\hline No & $18(20)$ & $6(19)$ & \\
\hline $\begin{array}{l}\text { EDSS at last available follow-up, } \\
\text { median (interval) }\end{array}$ & $7.5(0-10)$ & $6.0(1.5-10)$ & 0.16 \\
\hline Final outcome, $n(\%)$ & 93 & 25 & 0.48 \\
\hline Improved & 19 (21) & $7(28)$ & \\
\hline Stable & $15(16)$ & $4(16)$ & \\
\hline Worsened & 30 (32) & $10(40)$ & \\
\hline Death & 29 (31) & $4(16)$ & \\
\hline
\end{tabular}

Continued outcome. ${ }^{1}$ To limit infection in the brain, the rapid restoration of immunosurveillance is encouraged by interrupting the treatment and accelerating the removal of plasma NTZ, which is biologically active for up to 3 months after its infusion, with plasmapheresis (PLEX), which exchanges the plasma with donor plasma or albumin. Although the effectiveness of PLEX and the optimum treatment regimen have never been systematically investigated, PLEX is usually highly recommended in the management of NTZ-PML.,3 Although effective in removing NTZ, PLEX might be detrimental for patient outcome, increasing the likelihood of the development of immune reconstitution inflammatory syndrome (IRIS) and of inflammatory brain damage $^{4}$ from the rapid restoration of immunosurveillance.

In this study, we retrospectively analyzed 193 international NTZ-PML cases reported in the literature and 34 Italian NTZ-PML cases with the aim of estimating the risk/benefit profile of PLEX in NTZ-PML.

METHODS PubMed search. We searched PubMed for English articles that were published from January 2005 to June 2015 and that included the terms natalizumab and progressive multifocal leukoencephalopathy. We found 487 articles. Subsequently, we selected only those articles that reported clinical descriptions of the NTZ-PML patients, regardless of the original aim of the study. Articles lacking meaningful clinical information about the patients' PML diagnosis, treatments, and outcome, as well as articles about patients without MS (e.g., Crohn disease) who were treated with NTZ, were excluded. Patients described by multiple articles were listed once in the database with the most informative article listed as the source document. We excluded the following: cases and clinical series with suspected overlap between articles, previously published, Italian patients who were already included in the Italian dataset, and case series from which individual clinical information could not be extracted.

International published cases. A final sample of 193 international NTZ-PML cases, collected from 49 articles, ${ }^{\text {el- }-44,5-9}$ was included in this study. All of the cases fulfilled the PML diagnostic criteria suggested by Berger et al. ${ }^{10}$

For each case, we extracted the following demographic and clinical information: sex (male/female), age at time of PML diagnosis (years), country (Europe, United States, or rest of the world [ROW]), Expanded Disability Status Scale (EDSS) score prior to PML diagnosis, prior immunosuppressant exposure (yes/no), number of NTZ infusions at time of PML diagnosis, presence of symptoms at PML diagnosis (yes/no), PML lesion localization at diagnosis (supratentorial/infratentorial/both), CSF-JCV status at diagnosis (positive/negative), number of CSF-JCV copies (anytime; i.e., first CSF-JCV-positive PCR), PLEX treatment (yes/ no), additional treatments (mefloquine, mirtazapine, both, none), PML-IRIS development (yes/no), interval between PML 
Table 1 Continued

PLEX+ $(n=184)$

PLEX- $(\mathrm{n}=35)$

p Value

Death, $\mathrm{n}(\%)$

Yes

$29(16)$

4 (11)

No

$152(84)$

Follow-up time, mo, median (interval)'
31 (89)

$12(<1-26)$

Abbreviations: EDSS $=$ Expanded Disability Status Scale; IRIS = immune reconstitution inflammatory syndrome; JCV = JC virus; NTZ = natalizumab; PLEX = plasmapheresis; $\mathrm{PML}=$ progressive multifocal leukoencephalopathy; $\mathrm{ROW}=$ rest of the world.

The percentages were estimated from the number of available observations.

a Significant.

${ }^{b}$ The number of available observations for PLEX+/PLEX -: 182/35.

${ }^{\mathrm{c}}$ The number of available observations for PLEX+/PLEX-: 54/20.

${ }^{d}$ The number of available observations for PLEX+/PLEX -: 183/34.

${ }^{\mathrm{e}}$ The number of available observations for PLEX+/PLEX-: 40/16.

${ }^{f}$ The number of available observations for PLEX+/PLEX-: 168/30.

9 The number of available observations for PLEX+/PLEX-: 52/6.

${ }^{h}$ The number of available observations for PLEX+/PLEX -: 66/20.

iThe number of available observations for PLEX+/PLEX-: 72/25.

diagnosis and IRIS development (days), use of steroids for the treatment/prevention of IRIS (yes/no), clinical status at last examination available after PML resolution, and post-PML follow-up (months). PML-IRIS development and clinical status after PML were defined according to the authors' statements.

Italian cases. Thirty-four Italian NTZ-PML patients were included in this study. The data were retrospectively collected by Dr. Capra and collaborators from 25 Italian MS centers that were allowed to prescribe NTZ in accordance with the regulations of the Italian authorities. The majority $(n=20)$ of

\section{Figure 1 Clinical severity of natalizumab-progressive multifocal leukoence-} phalopathy $(\mathrm{PML})$ at diagnosis

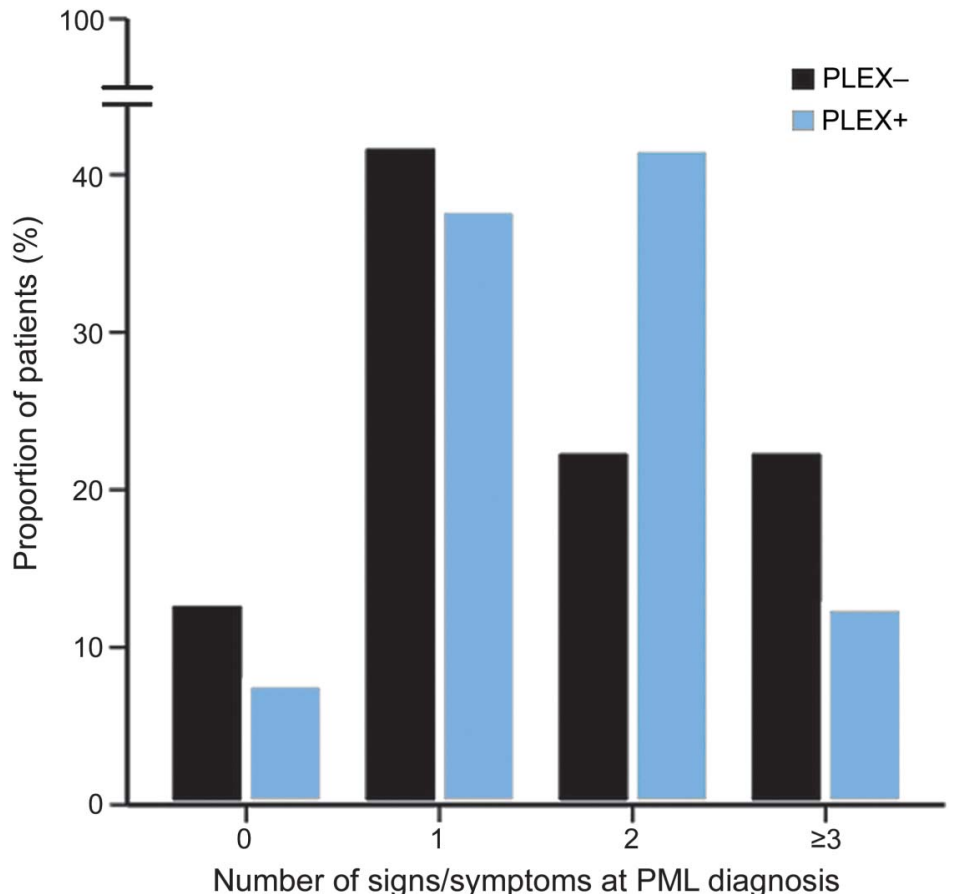

Clinical severity of PML (estimated as number of signs/symptoms) at diagnosis. PLEX = plasmapheresis.

the centers reported only 1 NTZ-PML patient, and only 1 site reported 4 NTZ-PML patients. In order to confirm the NTZPML diagnoses, the clinical and radiologic data sent by each center were reanalyzed. The same diagnostic criteria for NTZPML as those cited above were applied. For the Italian cases, the same clinical variables as those described for the international cases were collected.

Standard protocol approvals, registrations, and patient consents. International published cases. Because we analyzed data that were obtained from published reports, neither ethics committee approval nor informed consent was required.

Italian cases. The ethics committee of the Spedali Civili of Brescia approved the retrospective analysis of the patient data.

Outcome variables. For the primary outcome measures, we examined survival and post-PML clinical status. Nonsurvivors were patients who died at any time due to PML complications after being diagnosed with PML (e.g., 1 Italian patient died because of cholecystitis that was unrelated to the PML, and, therefore, he was considered a survivor). The post-PML clinical status was compared to the PML clinical status at the diagnosis and rated as stable, improved, worsened, or dead based on the reported clinical descriptions, EDSS/Karnofsky Performance Scale scores, or authors' statements. The post-PML neurologic evaluation that was last available at the time of the writing of each article was used. Any stated change in disability was considered a clinical impairment/ worsening, regardless of the magnitude of the effect.

The median interval of follow-up (months) between PML diagnosis and the last available evaluation or outcome reached (e.g., death), whichever came first, is specified in table 1. For the Italian cohort, the PML clinical outcome was assessed at 12 months.

Statistical analysis. The descriptive statistics are expressed as count (percentage) for discrete variables and mean (SD) or median (range) for continuous variables. The cohort was divided into 2 groups (PLEX+ and PLEX-) according to PLEX administration. The differences between the patients who underwent PLEX and those who did not were explored with $\chi^{2}$ tests or Mann-Whitney $U$ tests for categorical or continuous variables, respectively. Patients who were administered immunoadsorption were included in the PLEX+ group.

To assess the effects of PLEX and the demographic, clini$\mathrm{cal}$, and treatment variables on outcome after PML, univariate and multivariate Cox proportional hazards models were constructed with the 2 different outcomes, namely, overall mortality and the 4 ratings defining the patients after PML as improved or stable vs worsened or dead, considered the dependent variables. The demographic and clinical covariates that were associated with the aforementioned clinical outcomes with $p$ values $\leq 0.10$ in the univariate analysis were included in the multivariate models that included PLEX as the independent variable of interest. The multivariate models were corrected for sex and age.

Because the main objective of the present study was to analyze the association between PLEX treatment and outcome, PLEX was included in the multivariate models even if it was not statistically significant in the univariate analyses. The number of CSFJCV copies was log-transformed due to its positively skewed distribution.

The primary research question was to evaluate whether PLEX improves survival and clinical outcomes in patients with NTZPML.

Level of evidence. This study provides Class III evidence that for patients with NTZ-PML, PLEX does not improve survival. 

Table 2 Univariate and multivariate Cox hazard model analyses (using months of follow-up as the time
variable) assessing the effects of plasmapheresis (PLEX) and the demographic and clinical
variables on mortality after progressive multifocal leukoencephalopathy (PML) (events included in
the analysis $=9$ )

\begin{tabular}{|c|c|c|c|c|c|c|c|}
\hline \multirow[b]{2}{*}{ Variables } & \multirow[b]{2}{*}{ No. } & \multicolumn{3}{|c|}{ Univariate analysis } & \multicolumn{3}{|c|}{ Multivariate analysis } \\
\hline & & HR & $95 \% \mathrm{Cl}$ & p Value & HR & $95 \% \mathrm{Cl}$ & $p$ Value \\
\hline \multicolumn{8}{|l|}{ PLEX } \\
\hline No & 25 & 1.00 & - & - & 1.00 & - & - \\
\hline Yes & 72 & 1.25 & $0.40-3.92$ & 0.70 & 0.30 & $0.06-2.70$ & 0.14 \\
\hline \multicolumn{8}{|l|}{ Sex } \\
\hline Female & 75 & 1.00 & - & - & & & \\
\hline Male & 22 & 1.90 & $0.65-5.58$ & 0.24 & & & \\
\hline \multicolumn{8}{|l|}{ Age at PML diagnosis } \\
\hline Each year & 97 & 1.04 & $0.98-1.10$ & 0.20 & & & \\
\hline \multicolumn{8}{|l|}{ Country } \\
\hline Europe & 80 & 1.00 & - & - & 1.00 & - & - \\
\hline US + ROW & 17 & 21.25 & $6.95-64.96$ & $<0.001^{a}$ & 5.78 & $0.97-34.36$ & $0.05^{\mathrm{a}}$ \\
\hline \multicolumn{8}{|l|}{ Pre-PML EDSS } \\
\hline Each step & 72 & 0.86 & $0.56-1.31$ & 0.47 & & & \\
\hline \multicolumn{8}{|l|}{ No. of NTZ infusions } \\
\hline Each unit & 95 & 0.99 & $0.95-1.03$ & 0.57 & & & \\
\hline \multicolumn{8}{|c|}{ Prior immunosuppressants } \\
\hline No & 60 & 1.00 & - & - & & & \\
\hline Yes & 24 & 0.95 & $0.29-3.06$ & 0.93 & & & \\
\hline \multicolumn{8}{|c|}{ EDSS at PML diagnosis } \\
\hline Each step & 56 & 0.94 & $0.61-1.46$ & 0.80 & & & \\
\hline \multicolumn{8}{|l|}{ Asymptomatic PML } \\
\hline No & 11 & 1.00 & - & - & & & \\
\hline Yes & 81 & 0.04 & $0.01-43.29$ & 0.37 & & & \\
\hline \multicolumn{8}{|c|}{$\begin{array}{l}\text { PML lesion localization } \\
\text { at diagnosis }\end{array}$} \\
\hline Supratentorial & 67 & 1.00 & - & - & & & \\
\hline Infratentorial & 7 & 1.00 & 0.99-1.01 & 0.99 & & & \\
\hline Both & 9 & 2.01 & $0.41-9.82$ & 0.39 & & & \\
\hline \multicolumn{8}{|l|}{ CSF JCV status } \\
\hline Negative & 15 & 1.00 & - & - & & & \\
\hline Positive & 80 & 0.66 & $0.18-2.37$ & 0.53 & & & \\
\hline \multicolumn{8}{|l|}{ CSF JCV copies } \\
\hline Each log increase & 86 & 1.74 & $1.12-2.73$ & $0.01^{\mathrm{a}}$ & 1.29 & $0.06-2.70$ & 0.49 \\
\hline \multicolumn{8}{|l|}{ Additional treatments } \\
\hline No & 43 & 1.00 & - & - & & & \\
\hline Yes & 46 & 1.16 & $0.72-1.87$ & 0.53 & & & \\
\hline \multicolumn{8}{|c|}{$\begin{array}{l}\text { Time from PML diagnosis } \\
\text { to IRIS }\end{array}$} \\
\hline Each day & 36 & 0.98 & $0.95-1.02$ & 0.33 & & & \\
\hline \multicolumn{8}{|c|}{ PML-IRIS development } \\
\hline No & 20 & 1.00 & - & - & & & \\
\hline Yes & 72 & 29.70 & $0.10-1.03 \times 10^{4}$ & 0.26 & & & \\
\hline
\end{tabular}

Continued 
Table 2 Continued

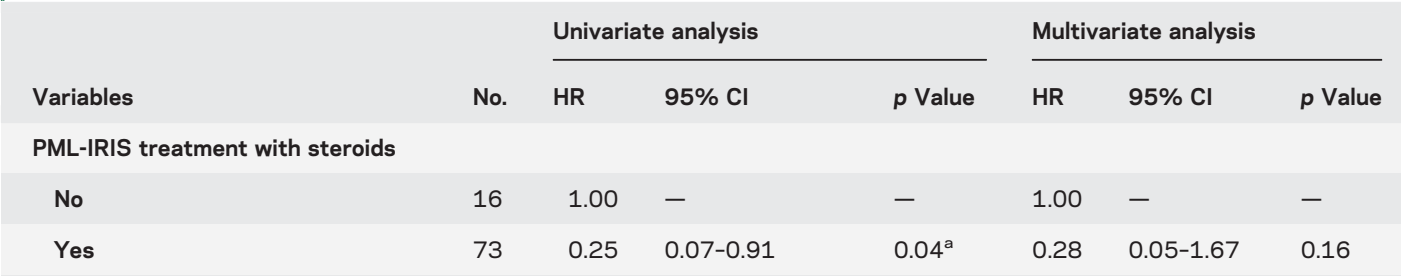

Abbreviations: $\mathrm{Cl}=$ confidence interval; EDSS = Expanded Disability Status Scale; HR = hazard ratio; IRIS = immune reconstitution inflammatory syndrome; JCV = JC virus; NTZ = natalizumab; ROW = rest of the world.

a Significant.

The study lacks the statistical precision to exclude an important benefit or harm of PLEX.

RESULTS After pooling the international and Italian cases, we analyzed the data for 227 NTZ-PML patients (56 from the United States and ROW, 137 from Europe, and 34 from the Italian cohort). Eight patients were excluded from the final analysis because the PLEX data were missing, which resulted in a final sample of 219 patients. The demographic and clinical characteristics of the patients are summarized in table 1. All of the patients stopped the NTZ treatment immediately following the PML diagnosis. PLEX was performed in 184 (84\%) of the 219 patients. The median number of PLEX courses (based on the available data for 30 patients) was 5 , with a range of 1 to 20 ( 5 courses repeated 4 times every 6 months). The 2 groups (PLEX+ and PLEX-) significantly differed for sex $(p=0.02)$, CSF-JCV copies $(p=$ $0.001)$, use of additional treatments $(p=0.002)$, and PML lesion localization $(p=0.04)$, as shown in table 1 . The clinical severity of the PML at diagnosis did not differ between the PLEX + and PLEX - groups $(p=0.22$; see figure 1$)$. There was no between-group difference for the follow-up intervals from the time of PML diagnosis $(p=0.20)$ (table 1$)$.

The univariate analysis showed that mortality (hazard ratio [HR] 1.25; confidence interval 0.40 3.92; $p=0.7$; table 2) and the likelihood of improving or remaining stable compared to worsening or dying (HR 1.25; $p=0.47$; table 3) did not differ between the patients who were treated with PLEX and those who were not. In addition, Kaplan-Meier survival curves for overall survival and time to outcome did not differ between the 2 groups $(p>0.4$ with the log-rank test; figure 2). Country (United States/ROW vs Europe) was a risk factor for both mortality (HR 21.25; $p<0.001$, table 2) and poor outcome (HR 5.70; $p<0.001$, table 3). The development of PML-IRIS was also associated with an increased risk of poor outcome (HR 6.35; $p=$ 0.01 , table 3) but not with mortality. The number of CSF-JCV copies was associated with a higher risk of mortality (HR 1.74; $p=0.01$, table 2 ). Moreover, the univariate Cox hazard model analysis showed that the treatment of PML-IRIS with steroids was a significant contributor to reduced mortality (HR 0.25; $p=$ 0.04, table 2).

The multivariate Cox hazard model analysis of mortality after PML found that country was the only predictive variable (HR 5.78; $p=0.05$, table 2). Country (HR 3.87; $p=0.001$, table 3) and PMLIRIS development (HR 4.61; $p=0.04$, table 3 ) were also predictive of poor outcome in the multivariate model.

DISCUSSION In recent years, PML has gained renewed attention due to reports of patients with MS who were treated with the monoclonal antibody NTZ. Because effective treatments for PML are not available, the rapid reconstitution of immune surveillance in the CNS through PLEX has been widely encouraged in order to limit viral spread. ${ }^{2,3}$ Adherence to this recommendation has been high worldwide, as was demonstrated by the population data gathered in this study. About 84\% (184 of 219 cases) of the entire NTZ-PML cohort had been treated with PLEX, while only $16 \%$ had not. The reasons why PLEX was not administered differed in each instance (e.g., long interval between PML diagnosis and NTZ withdrawal not requiring NTZ removal, ${ }^{8}$ first occurrences as adverse events in clinical trials, ${ }^{5,6}$ and lesion localization in eloquent areas, making the risk of PML-IRIS unjustifiable ${ }^{11}$ ). This imbalance in treatment approach confirmed that PLEX was considered the treatment of choice for NTZ-PML in clinical practice, even if clinical trials supporting its efficacy in this population are lacking.

To fill this gap, we compared the clinical outcomes of patients with MS and NTZ-PML who were treated with PLEX with those who were not in the present study. We did not find improvement in mortality or residual disability in patients treated with PLEX compared to the untreated patients. These findings suggested that the spontaneous recovery of immunocompetence after NTZ withdrawal might counteract the spread of PML in patients with MS 

Table 3 Univariate and multivariate Cox hazard model analysis (using months of follow-up as the time
variable) assessing the effects of plasmapheresis (PLEX) and the demographic and clinical variables
on the likelihood of poor vs favorable outcomes (i.e., worsened/dead vs improved/stable)

\begin{tabular}{|c|c|c|c|c|c|c|c|}
\hline \multirow[b]{2}{*}{ Variable } & \multirow[b]{2}{*}{ No. } & \multicolumn{3}{|c|}{ Univariate analysis } & \multicolumn{3}{|c|}{ Multivariate analysis } \\
\hline & & HR & $95 \% \mathrm{Cl}$ & p Value & HR & $95 \% \mathrm{Cl}$ & $p$ Value \\
\hline \multicolumn{8}{|l|}{ PLEX } \\
\hline No & 22 & 1.00 & - & - & 1.00 & - & - \\
\hline Yes & 44 & 1.25 & $0.68-2.32$ & 0.47 & 1.00 & $0.48-2.08$ & 0.99 \\
\hline \multicolumn{8}{|l|}{ Sex } \\
\hline Female & 70 & 1.00 & - & - & & & \\
\hline Male & 20 & 1.49 & $0.77-2.87$ & 0.23 & & & \\
\hline \multicolumn{8}{|c|}{ Age at PML diagnosis } \\
\hline Each year & 90 & 1.02 & $0.99-1.06$ & 0.17 & & & \\
\hline \multicolumn{8}{|l|}{ Country } \\
\hline Europe & 73 & 1.00 & - & - & 1.00 & - & - \\
\hline US + ROW & 17 & 5.70 & $2.98-10.93$ & $<0.001^{\mathrm{a}}$ & 3.87 & $1.78-8.42$ & $0.001^{a}$ \\
\hline \multicolumn{8}{|l|}{ Pre-PML EDSS } \\
\hline Each step & 70 & 0.93 & $0.78-1.11$ & 0.45 & & & \\
\hline \multicolumn{8}{|l|}{ No. of NTZ infusions } \\
\hline Each unit & 88 & 0.99 & $0.98-1.02$ & 0.68 & & & \\
\hline \multicolumn{8}{|c|}{ Prior immunosuppressants } \\
\hline No & 57 & 1.00 & - & - & & & \\
\hline Yes & 24 & 0.69 & $0.35-1.36$ & 0.28 & & & \\
\hline \multicolumn{8}{|c|}{ EDSS at PML diagnosis } \\
\hline Each step & 56 & 0.96 & $0.81-1.14$ & 0.68 & & & \\
\hline \multicolumn{8}{|l|}{ Asymptomatic PML } \\
\hline No & 11 & 1.00 & - & - & & & \\
\hline Yes & 78 & 0.86 & $0.36-2.03$ & 0.73 & & & \\
\hline \multicolumn{8}{|c|}{$\begin{array}{l}\text { PML lesion localization } \\
\text { at diagnosis }\end{array}$} \\
\hline Supratentorial & 64 & 1.00 & - & - & & & \\
\hline Infratentorial & 5 & 1.26 & $0.38-4.11$ & 0.70 & & & \\
\hline Both & 9 & 1.11 & $0.43-2.87$ & 0.82 & & & \\
\hline \multicolumn{8}{|l|}{ CSF JCV status } \\
\hline Negative & 14 & 1.00 & - & - & & & \\
\hline Positive & 74 & 0.67 & $0.32-1.37$ & 0.27 & & & \\
\hline \multicolumn{8}{|l|}{ CSF JCV copies } \\
\hline Each log-increase & 79 & 1.24 & $0.95-1.61$ & 0.10 & 1.10 & $0.82-1.45$ & 0.53 \\
\hline \multicolumn{8}{|c|}{ Additional treatments } \\
\hline No & 37 & 1.00 & - & - & & & \\
\hline Yes & 45 & 1.05 & $0.58-1.92$ & 0.87 & & & \\
\hline \multicolumn{8}{|c|}{ PML-IRIS development } \\
\hline No & 19 & 1.00 & - & - & 4.61 & $1.10-19.39$ & $0.04^{\mathrm{a}}$ \\
\hline Yes & 66 & 6.35 & $1.54-26.25$ & $0.01^{a}$ & & & \\
\hline \multicolumn{8}{|c|}{ Treatment with steroids } \\
\hline No & 16 & 1.00 & - & - & & & \\
\hline
\end{tabular}

Continued 
Table 3 Continued

\begin{tabular}{|c|c|c|c|c|c|c|c|}
\hline Variable & No. & HR & $95 \% \mathrm{Cl}$ & $p$ Value & HR & $95 \% \mathrm{Cl}$ & $p$ Value \\
\hline Yes & 66 & 0.65 & $0.32-1.35$ & 0.25 & & & \\
\hline
\end{tabular}

Abbreviations: $\mathrm{Cl}=$ confidence interval; EDSS = Expanded Disability Status Scale; HR = hazard ratio; IRIS = immune reconstitution inflammatory syndrome; JCV = JC virus; NTZ = natalizumab; PML = progressive multifocal leukoencephalopathy; ROW = rest of the world.

a Significant.

and therefore not require any additional intervention. Nevertheless, forcing the rapid restoration of immune surveillance in the brain with PLEX may eventually expose patients to an increased risk of aggressive PML-IRIS.7 In PML, the primary infection occurs in oligodendrocytes, which leads to oligodendrocyte death and demyelination. PML-IRIS develops when a massive amount of immunocompetent cells enter the CNS and localize within and at the border of visible PML lesions, where many infected oligodendrocytes are in a preapoptotic phase. These cells are the target of T-cell-mediated cytotoxicity that is elicited by immunoreconstitution and results in damage to surrounding tissues and clinical worsening. In addition, inflammation activates effector macrophages and microglia, further amplifying the inflammatory reaction and prompting blood-brain barrier damage, brain injury, and cerebral edema. ${ }^{9}$ This immunopathologic cascade is responsible for the devastating effects that lead to great clinical disability and possibly death.

In the present study, we confirmed the negative prognosis of PML-IRIS. It is now widely accepted that the more limited the infection and earlier the diagnosis, the milder the PML-IRIS and the better the postinfectious neurologic status. ${ }^{12}$ In our cohort, we identified 13 asymptomatic patients, and 8 of 12 were treated with PLEX, while 4 were not (information was missing for 1 patient). In this group, 5 of the treated patients remained asymptomatic, while 3 worsened, and all of the untreated (4 of 4) patients worsened. No one died. Therefore, even if the overall analysis of our cohort had shown no positive effects of PLEX on patient outcome, and the small number of asymptomatic patients did not allow for a definitive conclusion, we cannot rule out the hypothesis that the benefits of PLEX may outweigh the risks in a subgroup of patients in which the immune restoration induced by PLEX is protective and not destructive. Conversely, a slower recovery of immunocompetence following NTZ withdrawal might be a more promising strategy in patients with widespread infection at the time of diagnosis, while considering the increased risks associated with PLEX in advanced cases ${ }^{13}$ and the long interval between oligodendrocyte infection and lysis.

In accordance with the findings of Dong-Si et al., ${ }^{1}$ we found that older age and country of origin, namely the United States and ROW, were independently associated with poor outcome and survival. Because PLEX is equally available and applied across countries, other factors that were

Figure 2 Natalizumab-progressive multifocal leukoencephalopathy (PML) outcomes
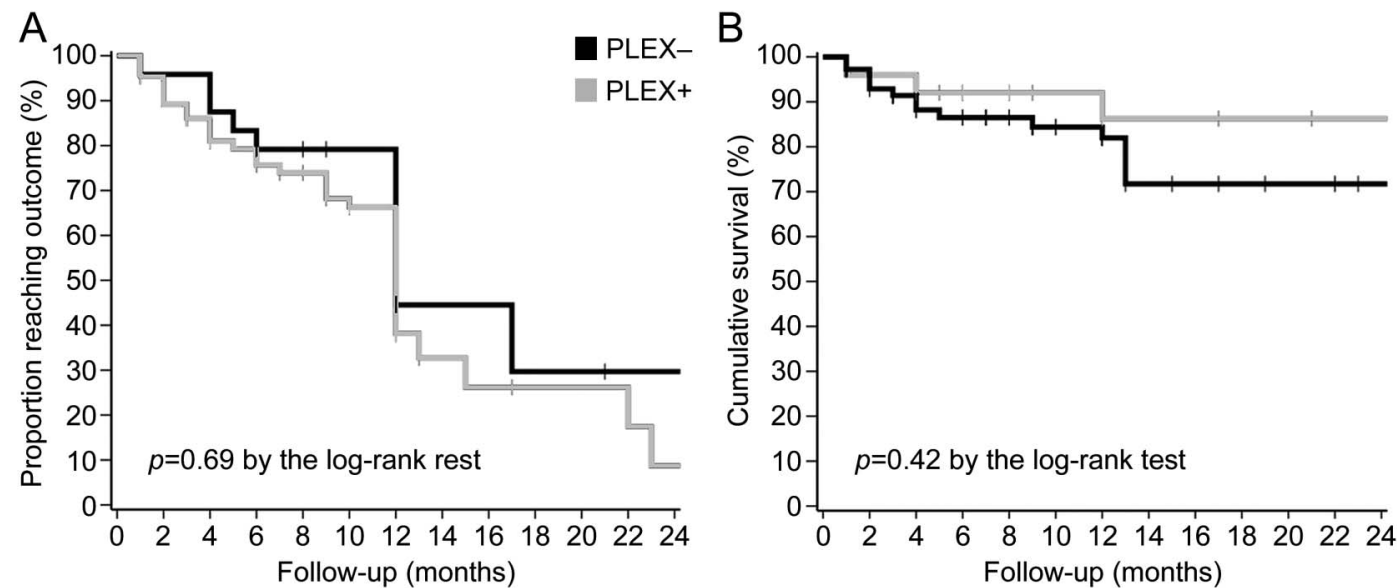

Kaplan-Meier curves show time to outcome (worsening or death, A) and overall survival (B) after PML diagnosis. PLEX = plasmapheresis. 
not evaluated in this study (e.g., time to diagnosis) might explain these results.

The current study had several limitations. The main limitations were related to the retrospective structure of the analysis and the indirect collection of the clinical measures derived from the published cases. Moreover, because the datasets were created by gathering information from studies that did not have the same aim as that of the present study, the data were often incomplete (i.e., data about PLEX regimen, type of PLEX, interval between NTZ withdrawal and PML diagnosis or PLEX administration, time to death, magnetic resonance lesion volume or specific lesion location description, and neurologic status at PML-IRIS development). In our cohort, due to the limited information available, we quantified PML-IRIS as a dichotomous variable (PML-IRIS: yes/no). Therefore, we could not estimate whether PMLIRIS was more severe in patients who were treated with PLEX, which should be addressed in future studies. In addition, the need to extract meaningful clinical information led us to exclude many articles from the analysis, thus creating a potential selection bias.

Notwithstanding these limitations, our study failed to show an effect of PLEX on improving clinical outcome and survival in Italian and international patients with MS who were diagnosed with NTZ-PML. Considering the potential risks and costs of PLEX, these results argue for caution and for individualized decision-making regarding PLEX. The clinical relevance of this problem justifies the performance of prospective clinical studies in order to identify the patients who will more likely benefit from PLEX in NTZ-PML treatment.

\section{AUTHOR CONTRIBUTIONS}

Doriana Landi: drafting of the manuscript content, including medical content; study concept or design; analysis or interpretation of data; acquisition of data. Nicola De Rossi: drafting of the manuscript content, including medical content; study concept or design; analysis or interpretation of data; acquisition of data. Sara Zagaglia: drafting of the manuscript content; analysis or interpretation of data; acquisition of data. Cristina Scarpazza: drafting of the manuscript content; acquisition of data. Luca Prosperini: statistical analysis, revising of the manuscript content. Maria Albanese: analysis or interpretation of data. Fabio Buttari: analysis or interpretation of data. Francesco Mori: analysis or interpretation of data. Girolama Alessandra Marfia: analysis or interpretation of data. Maria Pia Sormani: drafting/revising of the manuscript content; statistical analysis. Ruggero Capra: revising of the manuscript content; study concept or design; study supervision or coordination. Diego Centonze: revising of the manuscript content; study concept or design; study supervision or coordination.

\section{STUDY FUNDING}

No targeted funding reported.

\section{DISCLOSURE}

D. Landi has received travel funding from Novartis, Teva, Merck Serono, Almirall, Biogen, and Genzyme and is a subinvestigator in clinical trials being conducted for Novartis, Merck Serono, Teva, Biogen, and Roche. N. De Rossi reports no disclosures relevant to the manuscript. S. Zagaglia has received travel funding from Novartis, Teva, Merck Serono, Almirall, Biogen, and Genzyme and is a subinvestigator in clinical trials being conducted for Novartis, Merck Serono, Teva, Biogen, and Roche. C. Scarpazza reports no disclosures relevant to the manuscript. L. Prosperini has received consulting fees from Biogen and Novartis; speaker honoraria from Biogen, Genzyme, Novartis, and Teva; travel grants from Biogen, Genzyme, Novartis, and Teva; and research grants from the Italian MS society (Associazione Italiana Sclerosi Multipla) and Genzyme. M. Albanese has received travel funding from Novartis, Teva, Merck Serono, Almirall, Biogen, and Genzyme and is a subinvestigator in clinical trials being conducted for Novartis, Merck Serono, Teva, Biogen, and Roche. F. Buttari has received travel funding from Novartis, Teva, Merck Serono, Almirall, and Biogen. F. Mori reports no disclosures relevant to the manuscript. G. Marfia has received travel funding from Teva, Genzyme, and Biogen and is a principal investigator in clinical trials conducted for Novartis, Merck Serono, Teva, Biogen, and Roche. M. Sormani has received consulting fees from Biogen, Teva, Genzyme, Merck Serono, Novartis, Roche, and Vertex. R. Capra has received consulting fees from Biogen, Teva, Genzyme, Merck Serono, and Novartis. D. Centonze is an Advisory Board member of Merck-Serono, Teva, Bayer Schering, Biogen, Novartis, Almirall, GW Pharmaceuticals, Genzyme, and Roche, and he has received travel funding and honoraria for speaking or consultation fees from Merck Serono, Teva, Novartis, Bayer Schering, Sanofi-Aventis, Biogen, Almirall, and Genzyme. Go to Neurology.org for full disclosures.

Received July 20, 2016. Accepted in final form October 23, 2016.

\section{REFERENCES}

1. Dong-Si T, Gheuens S, Gangadharan A, et al. Predictors of survival and functional outcomes in natalizumabassociated progressive multifocal leukoencephalopathy. J Neurovirol 2015;21:637-644.

2. Ghezzi A, Grimaldi LM, Marrosu MG, et al. Natalizumab therapy of multiple sclerosis: recommendations of the multiple sclerosis study group-Italian neurological society. Neurol Sci 2011;32:351-358.

3. Khatri BO, Man S, Giovannoni G, et al. Effect of plasma exchange in accelerating natalizumab clearance and restoring leukocyte function. Neurology 2009;72:402-409.

4. Tan IL, McArthur JC, Clifford DB, Major EO, Nath A. Immune reconstitution inflammatory syndrome in natalizumab-associated PML. Neurology 2011;77: 1061-1067.

5. Kleinschmidt-DeMasters BK, Tyler KL. Progressive multifocal leukoencephalopathy complicating treatment with natalizumab and interferon beta-1a for multiple sclerosis. N Engl J Med 2005;353:369-374.

6. Langer-Gould A, Atlas SW, Green AJ, Bollen AW, Pelletier D. Progressive multifocal leukoencephalopathy in a patient treated with natalizumab. N Engl J Med 2005; 353:375-381.

7. Clifford DB, De Luca A, Simpson DM, Arendt G, Giovannoni $G$, Nath A. Natalizumab-associated progressive multifocal leukoencephalopathy in patients with multiple sclerosis: lessons from 28 cases. Lancet Neurol 2010;9: 438-446.

8. Killestein J, Vennegoor A, van Golde AEL, Bourez RLJH, Wijlens MLB, Wattjes MP. PML-IRIS during fingolimod diagnosed after natalizumab discontinuation. Case Rep Neurol Med 2014;2014:307872.

9. Tan LA, Lopes DK. Surgical management of malignant cerebral edema secondary to immune reconstitution 
inflammatory syndrome from natalizumab-associated progressive multifocal encephalopathy. J Clin Neurosci 2015; 22:1669-1671.

10. Berger JR, Aksamit AJ, Clifford DB, et al. PML diagnostic criteria: consensus statement from the AAN Neuroinfectious Disease Section. Neurology 2013;80:1430-1438.

11. Cordioli C, De Rossi N, Rasia S, Lodoli G, Capra R. Early detection and favourable outcome of natalizumabrelated progressive multifocal leukoencephalopathy
(PML) in two multiple sclerosis patients. Neurol Sci 2015;36:489-491.

12. Dong-Si T, Richman S, Wattjes MP, et al. Outcome and survival of asymptomatic PML in natalizumab-treated MS patients. Ann Clin Transl Neurol 2014;1:755-764.

13. Bauer J, Gold R, Adams O, Lassmann H. Progressive multifocal leukoencephalopathy and immune reconstitution inflammatory syndrome (IRIS). Acta Neuropathol 2015;130:751-764.

\section{Get Connected. Stay Connected.}

Connect with the American Academy of Neurology's popular social media channels to stay up-todate on the latest news and breakthroughs in neurology, and network with peers and neurology thought leaders. Visit AAN.com/Connect.

\section{Seeking Established Neurologists! 2017 Transforming Leaders Program}

Are you an experienced US AAN member neurologist 10 or more years out of residency looking for a one-of-a-kind leadership experience that will help you lead at the AAN, in daily life, and in the world of neurology? The Transforming Leaders Program may be for you! Apply by June 1, 2017, at AAN.com/view/TransformingLeaders.

\section{Neurology ${ }^{\circledR}$ Genetics Call For Papers}

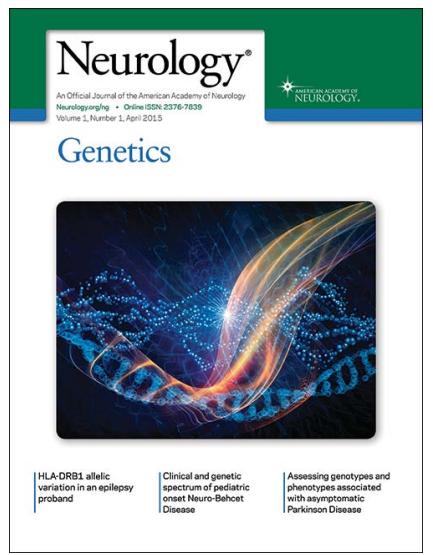

Neurology: Genetics is an open access, online only journal that provides neurologists with outstanding original contributions that elucidate the role of genetic and epigenetic variation in diseases and biological traits of the central and peripheral nervous system. We welcome all submissions. For more information on how to submit, visit http://www.neurology.org/site/ gen/gen2.xhtml. 